\title{
Optically induced dynamics of muonium centers in Si studied via their precession signatures
}

\author{
I. Fan, ${ }^{1}{ }^{*}$ K. H. Chow,,${ }^{1} \dagger$ B. Hitti, ${ }^{2}$ R. Scheuermann, ${ }^{3}$ W. A. MacFarlane, ${ }^{4}$ A. I. Mansour, ${ }^{1}$ B. E. Schultz, ${ }^{1}$ M. Egilmez, ${ }^{1}$ \\ J. Jung, ${ }^{1}$ and R. L. Lichti ${ }^{5}$ \\ ${ }^{1}$ Department of Physics, University of Alberta, Edmonton, Alberta, Canada T6G 2G7 \\ ${ }^{2}$ TRIUMF, 4004 Wesbrook Mall, Vancouver, British Columbia, Canada V6T $2 A 3$ \\ ${ }^{3}$ Paul Scherrer Institute, CH-5232 Villigen, PSI Switzerland \\ ${ }^{4}$ Department of Chemistry, University of British Columbia, Vancouver, British Columbia, Canada V6T $1 Z 1$ \\ ${ }^{5}$ Department of Physics, Texas Tech University, Lubbock, Texas 79409-1051, USA
}

(Received 20 September 2007; published 7 January 2008)

\begin{abstract}
We studied the influence of the optical excitation on three muonium centers $\mathrm{Mu}_{T}^{0}, \mathrm{Mu}_{B C}^{0}$, and $\mathrm{Mu}_{B C}^{+}$in high resistivity silicon. These investigations were carried out on the spin precession signature of each center as a function of temperature. It is found that photoexcitation resulted in significant enhancements of the depolarization rates of the precession signals as the three muonium centers underwent interactions with photogenerated free carriers. The results are described by a three-state model involving transitions between $\mathrm{Mu}_{T}^{0}, \mathrm{Mu}_{B C}^{0}$, and $\mathrm{Mu}_{B C}^{+}$as well as spin exchange processes.
\end{abstract}

DOI: 10.1103/PhysRevB.77.035203

PACS number(s): 76.75.+i, 72.20.Jv, 71.20.Mq

\section{INTRODUCTION}

Hydrogen $(\mathrm{H})$ is a technologically important impurity that dramatically modifies the electrical and optical properties of $\mathrm{Si}$ and many other semiconductors. ${ }^{1,2}$ This often occurs because hydrogen quickly passivates many types of defects in these materials, leading to both detrimental (e.g., passivation of shallow intentional dopants) and beneficial (e.g., passivation of dangling bonds) consequences. In certain semiconductors, $\mathrm{H}$ can even act as a shallow dopant. ${ }^{3}$ A complete understanding of the behavior of $\mathrm{H}$ in semiconductors requires a comprehensive knowledge of the role of isolated hydrogen, i.e., the precursor state in many situations, in the semiconductor. However, the rapid reactivity of hydrogen means that many of the techniques that are used to investigate hydrogen in semiconductors are not able to probe isolated $\mathrm{H}$. Studies of muonium $\left(\mathrm{Mu}^{0}=\mu^{+} e^{-}\right)$using the muon spin rotation/relaxation/resonance $(\mu \mathrm{SR})$ techniques are now widely recognized to be the main experimental source of information on isolated $\mathrm{H}$ in many semiconductors. ${ }^{4-23}$

Recall that the muon $\left(\mu^{+}\right)$is a radioactive particle with a lifetime of $\approx 2.2 \mu \mathrm{s}$. It can be considered a pseudoisotope of hydrogen with $\approx \frac{1}{9}$ th the mass of the proton, but is still much heavier than the electron $(\approx 200 \times)$. Hence, the electronic structures of $\mathrm{Mu}^{0}$ and $\mathrm{H}^{0}$ are very similar. However, the significantly different masses imply that certain processes, such as those involving diffusion, can be dramatically different. When $\mu^{+}$is implanted into high resistivity silicon, three muonium centers are formed. At low temperatures, two neutral muonium states have been experimentally identified, commonly labeled in the literature as $\mathrm{Mu}_{T}^{0}$ and $\mathrm{Mu}_{B C}^{0}$. The $\mathrm{Mu}_{T}^{0}$ center is believed to diffuse rapidly through the lattice via tetrahedral interstitial sites and, consequently, has an isotropic hyperfine interaction $(\approx 2000 \mathrm{MHz})$ that is approximately half that of muonium in vacuum. ${ }^{4}$ The $\mathrm{Mu}_{B C}^{0}$ state is located at the $\mathrm{Si}-\mathrm{Si}$ bond center (BC) and therefore has an anisotropic hyperfine interaction. ${ }^{24}$ This interaction is axially symmetric about a $\langle 111\rangle$ crystalline axis and is described by two parameters $A_{\|}$and $A_{\perp}$, which are approximately an order of magnitude smaller than the Fermi contact interaction ${ }^{25}$ of $\mathrm{Mu}_{T}^{0}$, i.e., $A_{\|}=-16.82 \mathrm{MHz}$ and $A_{\perp}=-92.59 \mathrm{MHz}$. At elevated temperatures, these neutral centers convert into the positively charged muonium center, $\mathrm{Mu}_{B C}^{+}{ }^{4}$ If a significant concentration of free electrons is present in the sample, such as in $n$-type $\mathrm{Si}$, additional dynamical processes involving the various muonium centers are observed. In one such process, often called spin exchange, the electron spin on $\mathrm{Mu}^{0}$ "flips" due to scattering with a free carrier. ${ }^{11,26}$ Another process is cyclic charge exchange (or charge-state fluctuation) ${ }^{27}$ whereby the charge state of a muonium center fluctuates between two states: For example, at sufficiently high temperatures, $\mathrm{Mu}^{0}$ ionizes but captures a free electron within its lifetime, and hence enters a cyclic charge changing process. Furthermore, if the electron concentration exceeds $\approx 10^{14} \mathrm{~cm}^{-3}$, a fourth muonium state $\mathrm{Mu}_{T}^{-}$can be formed. ${ }^{28}$

Instead of doping the $\mathrm{Si}$ with impurities, another method of introducing free charge carriers is by using optical excitation to athermally generate electron-hole pairs. Provided that muonium has time to undergo significant interactions with these carriers prior to its decay, photoexcitation experiments enable additional carrier capture processes, which would otherwise not take place in an unilluminated sample at a given temperature. Hence, this is a potentially powerful way to probe muonium dynamical processes involving interactions with free carriers.

Previous studies of muonium in $\mathrm{Si}$ (and other semiconductors) using photoexcitation have primarily been carried out ${ }^{29,30}$ at pulsed muon facilities using the conventional longitudinal field (LF)- $\mu \mathrm{SR}$ technique. ${ }^{5}$ By investigating the amplitude and $1 / T_{1}$ relaxation rates of the muon polarization, dynamics involving the three muonium states and the free carriers are implicitly observed. These dynamics include charge-state changes and site changes. The interpretation of such longitudinal field data is often complicated by the fact that the polarization consists of contributions from all the muonium states that are simultaneously present in the sample at that particular temperature, making it difficult to separate out the effects of optical excitation on a particular center. 
Moreover, the dynamics and the muonium center(s) involved in the interactions at a particular temperature can only be revealed by studying the amplitude and relaxation rates at many fields. This implies that conducting a detailed temperature-dependent study of the influence of light on the multiple muonium centers is a very time-consuming process and hence impractical given the limited access to $\mu$ SR facilities. Studies of photoexcited Si combined with radiofrequency (rf) measurements have also been reported. ${ }^{31}$ Only the behavior of the diamagnetic state was studied in these measurements. It was found that illumination has pronounced effects on the corresponding rf- $\mu \mathrm{SR}$ signal.

Another way to investigate photoexcited muonium dynamics is by monitoring the effects of light on the precession signatures of the muonium centers directly. Recall that since the hyperfine interactions of $\mathrm{Mu}_{B C}^{0}, \mathrm{Mu}_{T}^{0}$, and the diamagnetic center are all different, a unique set of precession frequencies is associated with each of the three states. This provides an unambiguous identification of the state that is involved. Furthermore, since coherent precession will only be observed if the particular muonium state is formed "promptly," the initial states can be selected out. Hence, such studies should complement those carried out with the LF- $\mu \mathrm{SR}$ and rf- $\mu \mathrm{SR}$ techniques. These experiments are best carried out at continuous muon facilities because the precession frequencies are often fast and hence not observable at pulsed muon facilities. ${ }^{5}$ In addition, at continuous muon facilities, higher relaxation rates can be investigated. The disadvantage, compared to experiments at pulsed muon facilities, is that the light should be intense enough to produce sufficient carriers for interactions with muonium, yet must not be so bright that significant heating of the sample occurs. Studies of the optically induced changes of the precession signals are sparse. An early study of the diamagnetic precession signature under photoexcitation has been carried out at low temperatures (below $\approx 40 \mathrm{~K}$ ) in doped Si. It was found the diamagnetic relaxation can be enhanced by illumination. ${ }^{32}$

In this paper, we demonstrate that the precession signatures of all the muonium states in silicon can indeed be strongly modified by optical excitation. In particular, the depolarization rates of the precession signals are dramatically enhanced, and their temperature dependence can be modeled by a three-state model involving transitions between $\mathrm{Mu}_{T}^{0}$, $\mathrm{Mu}_{B C}^{0}$, and $\mathrm{Mu}_{B C}^{+}$, provided that interactions with photogenerated carriers are incorporated.

\section{EXPERIMENTAL SETUP}

The optical excitation experiments described in this paper were carried out at the M15 and M20 beamlines at the TriUniversity Meson Facility (TRIUMF) located in Vancouver, Canada, where $\mu \mathrm{SR}, \beta$-NMR, ${ }^{33}$ and $\beta$-NQR ${ }^{34}$ experiments can be carried out. Positive muons with $\approx 100 \%$ polarization and nominal momentum $29.8 \mathrm{MeV} / \mathrm{c}$ were implanted into the sample. The sample was obtained from Siltronix, and is a single-side polished, float-zone, high resistivity $(10-30 \mathrm{k} \Omega \mathrm{cm})$, slightly boron doped silicon wafer with thickness of $600 \mathrm{~nm}$ and orientation $\langle 100\rangle$.

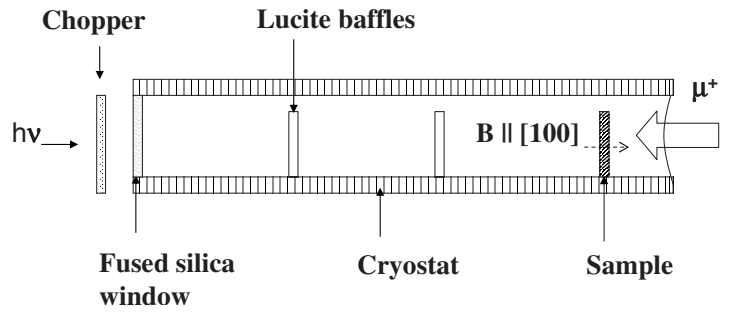

FIG. 1. Schematic diagram of the $\mu$ SR cryostat for the optical excitation experiment. The incoming $\mu^{+}$can be polarized to be either transverse or longitudinal to the applied magnetic field direction.

The schematic of the optical cryostat is summarized in Fig. 1. The sample was mounted on a thin lucite plate inside a horizontal helium gas flow cryostat with a transparent silica window on its backside. (Additional lucite baffles were present to disrupt the flow of helium and provide reasonable cooling efficiency.) A broad spectrum lamp (Gilway Technical L7390) was used to illuminate the (unpolished) side of the sample through the window, while the muons were implanted into the front (polished) side of the sample. The interior wall of the cryostat was covered with a highly reflective Mylar sheet to increase the amount of light that traveled down the length of the cryostat. The light beam was modulated on/off by placing a Boston Electronics SH-20-L-5 optical shutter in the path of the light (which was continuously on). The illumination on the sample is on for $1 \mathrm{~s}$, then off for $1 \mathrm{~s}$, with a $30 \mathrm{~ms}$ delay between the on/off states. Spectra with the light on and off were recorded in separate histograms. The relatively fast on/off modulation of the light minimized the temperature variations of the sample during the light on/off cycles. The temperature of the sample was monitored with a GaAlAs thermometer placed behind the sample. Positron and muon counters were arranged in standard configurations that were appropriate for LF- $\mu$ SR and transverse field (TF)- $\mu \mathrm{SR}$ measurements (see Ref. 5 for more details).

\section{PRECESSION SIGNATURES}

This paper focuses on the influence of optical excitation on the precession signatures of $\mathrm{Mu}_{B C}^{+}, \mathrm{Mu}_{B C}^{0}$, and $\mathrm{Mu}_{T}^{0}$. This section provides a reminder and/or overview of how these precession signatures can be obtained experimentally. All precession frequencies are intentionally chosen to reside in the window of 10-20 MHz.

The singly charged muonium center (such as $\mathrm{Mu}_{B C}^{+}$) is studied in a transverse field of $\approx 0.1 \mathrm{~T}$. Since no hyperfine interaction is associated with this center, the muon precesses at the Larmor frequency given by $\gamma_{u} H$, where the muon gyromagnetic ratio is $\gamma_{u}=135.54 \mathrm{MHz} / \mathrm{T}$ and $H$ is the magnitude of the applied field. An example of the spectra for $\mathrm{Mu}_{B C}^{+}$ is shown in Fig. 2(a). In the absence of illumination, there is very little relaxation of the muon polarization. Under illumination, a strong relaxation is observed [Fig. 2(b)].

The bond-centered muonium $\left(\mathrm{Mu}_{B C}^{0}\right)$ is studied in a longitudinal field of $\approx 0.2 \mathrm{~T}$ applied parallel to a $\langle 100\rangle$ direction 


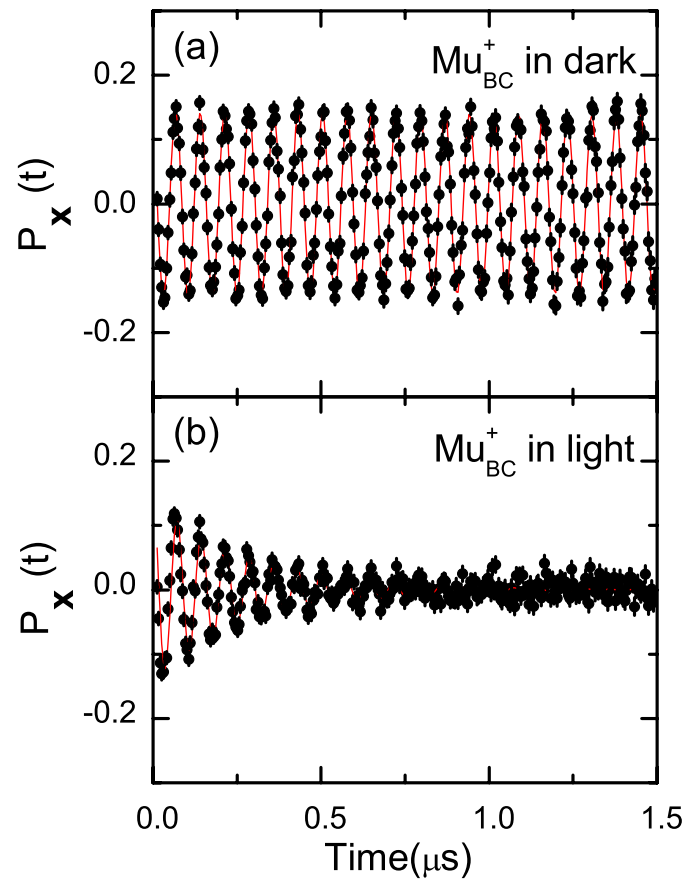

FIG. 2. (Color online) Typical TF- $\mu$ SR spectra for $\mathrm{Mu}_{B C}^{+}$in a field of $0.1 \mathrm{~T}$ and $\approx 260 \mathrm{~K}(\mathrm{a})$ in the dark and (b) under illumination. The circles are the experimental data and the solid lines are the fits as described in the text.

of the sample. At this magnetic field, about half of the muons can be thought of as precessing about an effective magnetic field that differs in both magnitude and direction from the applied field $\mathbf{H}$. Hence, although the initial muon spin is parallel to $\mathbf{H}$, a precessing signal of significant magnitude is observed. In addition, $0.2 \mathrm{~T}$ is close to a so-called "magic field," where the precession frequency (at $\approx 19.0 \mathrm{MHz}$ ) is essentially independent of the orientation between $\mathbf{H}$ and the $\mathrm{Mu}_{B C}^{0}$ hyperfine axis (also the bond axis). ${ }^{15,35}$ Hence, in the absence of any dynamics, the muon spin polarization should be long lived. This is indeed the case, as seen in Fig. 3(a), for $\mathrm{Mu}_{B C}^{0}$ at low temperatures. However, under optical excitation, there are significant changes in the spectrum: In particular, as shown in Fig. 3(b), the precession signal shows significant relaxation with a noticeable $1 / T_{1}$ component.

The muonium center $\mathrm{Mu}_{T}^{0}$ is characterized by an isotropic hyperfine parameter $A_{\|}=A_{\perp} \approx 2006 \mathrm{MHz}$ (at low temperatures) and is studied in a transverse field of $H \approx 1 \mathrm{mT}$. Recall that at low fields, two of the precession frequencies, commonly referred to as $\nu_{12}$ and $\nu_{23}$ in the literature, are given to a very good approximation by $\gamma_{e} H / 2$, where the electron gyromagnetic ratio $\gamma_{e}=28025 \mathrm{MHz} / \mathrm{T}$. In our experiment, as indicated in Fig. 4(a), the precession frequency for this center occurs at $\approx 11.9 \mathrm{MHz}$. Note that there is significant relaxation of the unilluminated $\mathrm{Mu}_{T}^{0}$ signal at all temperatures. Above $\approx 75 \mathrm{~K}$, illumination also produces relaxation of the $\mathrm{Mu}_{T}^{0}$ signal [see Fig. 4(b)].

The influence of optical excitation on the dynamics of the three muonium centers depends on the temperature of the sample and will be discussed in more detail in Sec. V. Nevertheless, it is qualitatively clear from Figs. 2-4 that there are dramatic differences in the muon polarization of all three

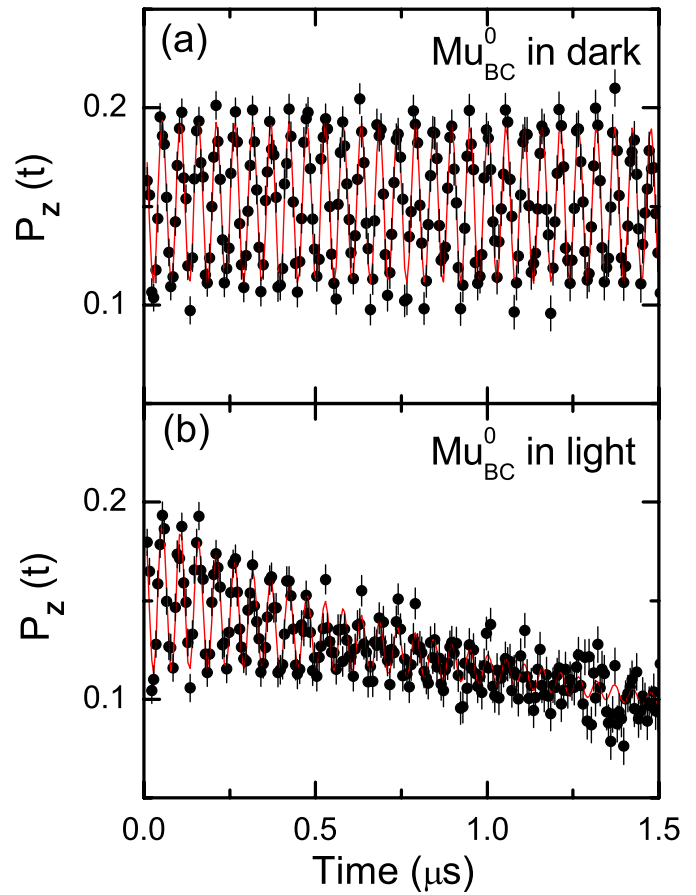

FIG. 3. (Color online) Typical LF- $\mu$ SR spectra for $\mathrm{Mu}_{B C}^{0}$ in a field of $\approx 0.2 \mathrm{~T}$ and $\approx 64 \mathrm{~K}$ (a) in the dark and (b) under illumination. The circles are the experimental data and the solid lines are the fits as described in the text.

centers in an illuminated sample compared to a sample in the dark. Hence, these results demonstrate that the precession signatures of the various muonium states in high resistivity Si can be dramatically affected by photoexcitation.

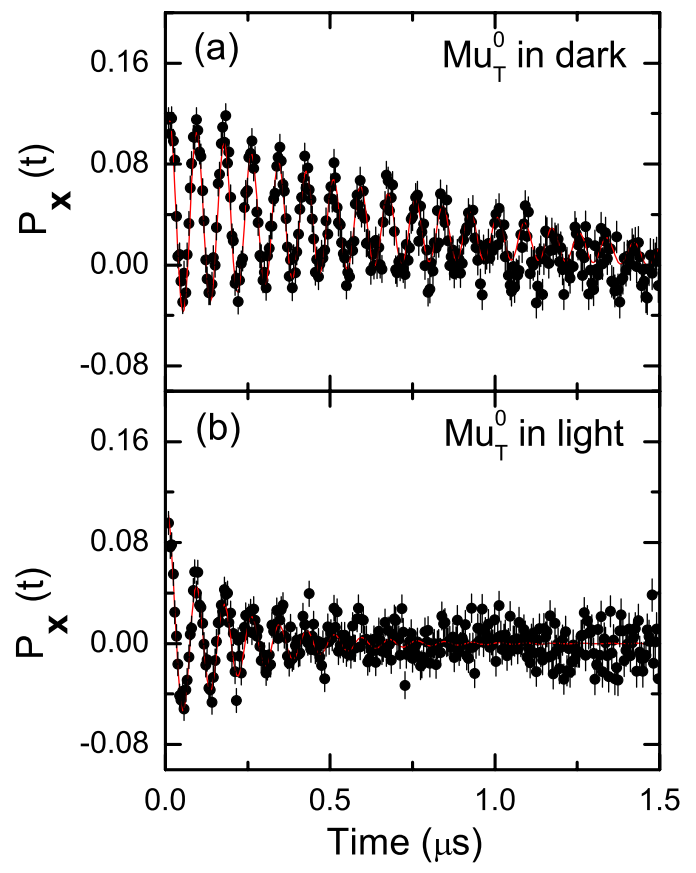

FIG. 4. (Color online) Typical TF- $\mu$ SR spectra for $\mathrm{Mu}_{T}^{0}$ in a field of $\approx 1 \mathrm{mT}$ and $\approx 269 \mathrm{~K}$ (a) in the dark and (b) under illumination. The circles are the experimental data and the solid lines are the fits as described in the text. 


\section{DATA ANALYSIS}

The quantities of interest that we extract from the precession data are the relaxation rates and the amplitudes associated with the three centers $\mathrm{Mu}_{B C}^{+}, \mathrm{Mu}_{B C}^{0}$, and $\mathrm{Mu}_{T}^{0}$. The experimental precession data for $\mathrm{Mu}_{B C}^{+}$, for which a typical spectrum is shown in Fig. 2, is well described by the function

$$
P_{x}(t)=A_{\mu} e^{-\lambda_{\mu} t} \cos \left(\omega_{\mu} t+\theta_{\mu}\right)
$$

where $A_{\mu}$ is the initial asymmetry, $\lambda_{\mu}$ is the relaxation rate, $\omega_{\mu}$ is the muon Larmor precession frequency, and $\theta_{\mu}$ is the phase of the signal.

The fitting function applied to the data associated with $\mathrm{Mu}_{B C}^{0}$, such as that shown in Fig. 3, is

$$
P_{z}(t)=A_{L F} e^{-\lambda_{L F} t}+A_{\mathrm{BC}} e^{-\lambda_{B C} t} \cos \left(\omega_{B C} t+\theta_{B C}\right) .
$$

The first term, characterized by initial asymmetry $A_{L F}$ and exponential relaxation $\lambda_{L F}$, becomes noticeable upon optical excitation of the sample. As discussed in Sec. III, a precession signal due to $\mathrm{Mu}_{B C}^{0}$ is observed in a longitudinal field experiment close to the magic field. This is parametrized by the second term, which is characterized by the initial amplitude $A_{B C}$, the exponential relaxation parameter $\lambda_{B C}$, the $\mathrm{Mu}_{B C}^{0}$ precession frequency $\omega_{B C}$, and the phase $\theta_{B C}$.

The fitting function used to model the $\mathrm{Mu}_{T}^{0}$ data, such as that shown in Fig. 4, is

$$
P_{x}(t)=A_{T} e^{-\lambda_{T} t} \cos \left(\omega_{T} t+\theta_{T}\right)+A_{2} e^{-\lambda_{2} t} \cos \left(\omega_{2} t+\theta_{2}\right)
$$

Since a precession signal due to $\mathrm{Mu}_{B C}^{+}$can also be observed in the applied transverse field of $1 \mathrm{mT}$, the first term is used to describe $\mathrm{Mu}_{T}^{0}$ while the second term describes $\mathrm{Mu}_{B C}^{+}$.

\section{RESULTS AND DISCUSSION}

The differences in the spectra observed under illumination and in the dark, such as those shown in Figs. 2-4, are attributed to interactions between muonium and the photogenerated carriers. The existence of these carriers enables processes that would otherwise be unimportant. In order to quantify the effects of optical excitation, we will examine the dynamics involved with the three muonium states $\mathrm{Mu}_{T}^{0}$, $\mathrm{Mu}_{B C}^{0}$, and $\mathrm{Mu}_{B C}^{+}$. The $\mathrm{Mu}_{T}^{-}$state is not included explicitly in our model since the maximum photoinduced carrier concentrations in this work remains below the threshold for a significant $\mathrm{Mu}_{T}^{-}$formation. The functional forms, as well as the parameter values characterizing the transitions that are relevant for an unilluminated sample within this three-state model, has been discussed by Kreitzman et al. ${ }^{8}$ and Hitti et al., ${ }^{28}$ i.e. in order to explain their rf- $\mu \mathrm{SR}$ (dark) Si data. In the analysis of our current data, we use the same functional forms for these transitions and also introduce additional processes that become important when the photoinduced carrier concentration becomes significant.

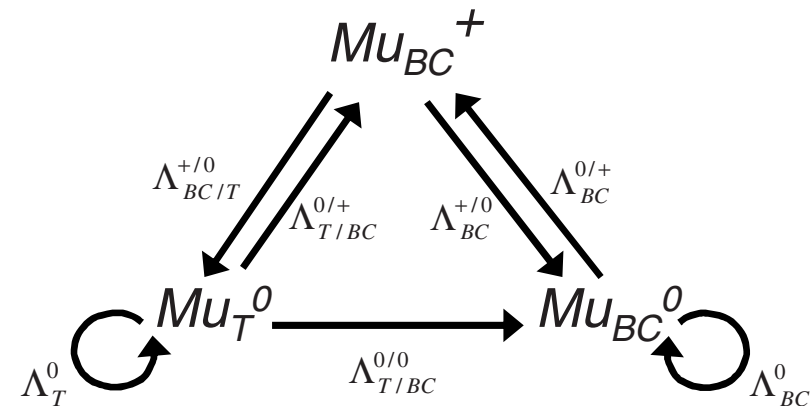

FIG. 5. The three-state model of muonium in Si under photoexcitation. The notation is described in more detail in the text.

This model is summarized in Fig. 5. As can be seen from Fig. 5, and as discussed in more detail below, we include the following dynamical interactions between $\mathrm{Mu}_{B C}^{+}, \mathrm{Mu}_{B C}^{0}$, and $\mathrm{Mu}_{T}^{0}$ : (i) Arrhenius thermally activated transitions, (ii) spin exchange processes involving $\mathrm{Mu}_{T}^{0}$ and $\mathrm{Mu}_{B C}^{0}$, (iii) capture processes of electrons and/or holes, which lead to changes in the charge state, and (iv) "activated capture" processes which take the site change into account. The transition rate is denoted as $\Lambda$ in the model, with the superscript representing the charge-state change and the subscript representing the site change. For example, a $0 /+$ superscript would indicate a transition of a neutral center to one that is positively charged, and a $\mathrm{BC} / T$ subscript would indicate that the muonium located at a BC site makes a transition to a $T$ site. The symbols $\Lambda_{T}^{0}$ and $\Lambda_{B C}^{0}$ in Fig. 5 denote spin exchange processes involving $\mathrm{Mu}_{T}^{0}$ and $\mathrm{Mu}_{B C}^{0}$, respectively.

The parameter values characterizing the dynamical interactions are summarized in Table I. They are inferred from simulations aimed at describing simultaneously the temperature dependences of the relaxation rates of all three muonium states. The simulated relaxation rates can be obtained by calculating the time evolution of the muon polarization within the dynamical model presented in Fig. 5; these are discussed in more detail in the Appendix.

Note that the initial amplitudes of the precession signatures of all three states are not affected by the optical excitation at any temperature, indicating that the photoexcitation does not change the implanted muon's initial state distribution significantly. [An example of this can be seen from the $\mathrm{Mu}_{B C}^{+}$amplitude in Fig. 2 as well as in the inset of Fig. 6(b).]

We now discuss in more detail the relaxation rates associated with each of the three muonium centers $\mathrm{Mu}_{B C}^{0}, \mathrm{Mu}_{B C}^{+}$, and $\mathrm{Mu}_{T}^{0}$. First, we address the $\mathrm{Mu}_{B C}^{0}$ center. As can be seen from Figs. 3 and $6(\mathrm{a})$, the $\mathrm{Mu}_{B C}^{0}$ precession signal shows little depolarization at low temperatures in the dark. However, there is an exponential increase in the dark relaxation rate beginning at $\approx 140 \mathrm{~K}$, which by $\approx 170 \mathrm{~K}$ is too fast to be observed. This is attributed to the thermal ionization of $\mathrm{Mu}_{B C}^{0}$ into $\mathrm{Mu}_{B C}^{+}$(i.e., $\left.\mathrm{Mu}_{B C}^{0} \rightarrow \mathrm{Mu}_{B C}^{+}+e^{-}\right){ }^{4,8,26}$ An Arrhenius relation

$$
\alpha_{B C}^{0 /+} \exp ^{\left(-E_{B C}^{0 /+} / k_{B} T\right)}
$$

$\left(\alpha_{B C}^{0 /+}\right.$ is the prefactor frequency and $E_{B C}^{0 /+}$ is the activation energy) can be used to characterize this ionization transition. 
TABLE I. Transitions identified by the optical excitation experiment and their values.

\begin{tabular}{|c|c|c|}
\hline \multicolumn{3}{|l|}{$\mathrm{Mu}_{B C}^{0}$ relaxation } \\
\hline Spin exchange & $\Lambda_{B C}^{0}=n v_{n} \sigma_{B C}^{0}$ & $\sigma_{B C}^{0}=2(1) \times 10^{-14} \mathrm{~cm}^{2}$ \\
\hline \multicolumn{3}{|l|}{ Charge exchange } \\
\hline $\mathrm{Mu}_{B C}^{0} \rightarrow \mathrm{Mu}_{B C}^{+}+e^{-}$ & $\Lambda_{B C}^{0 /+}=\alpha_{B C}^{0 /+} \exp ^{\left(-E_{B C}^{0 /+} / k_{B} T\right)}+p v_{p} \sigma_{B C}^{0 /+}$ & $\alpha_{B C}^{0 /+}=3.1(2) \times 10^{7} \mathrm{MHz}$ \\
\hline $\mathrm{Mu}_{B C}^{0}+h^{+} \rightarrow \mathrm{Mu}_{B C}^{+}$ & & $\begin{array}{l}E_{B C}^{0 /+}=0.21(1) \mathrm{eV} \\
\sigma_{B C}^{0 /+}=4(1) \times 10^{-15} \mathrm{~cm}^{2}\end{array}$ \\
\hline \multicolumn{3}{|l|}{$\mathrm{Mu}_{B C}^{+}$relaxation } \\
\hline \multicolumn{3}{|l|}{ Charge exchange } \\
\hline $\mathrm{Mu}_{B C}^{+}+e^{-} \rightarrow \mathrm{Mu}_{B C}^{0}$ & $\Lambda_{B C}^{+/ 0}=n v_{n} \sigma_{B C}^{+/ 0}$ & $\sigma_{B C}^{+/ 0}=3.3 \times 10^{-13} \mathrm{~cm}^{2}$ (fixed) \\
\hline \multirow[t]{3}{*}{$\mathrm{Mu}_{B C}^{+}+e^{-} \rightarrow \mathrm{Mu}_{T}^{0}$} & $\Lambda_{B C / T}^{+/ 0}=n v_{n} \sigma_{B C / T}^{+/ 0} \exp ^{\left(-E_{B C / T^{\prime}}^{\left.+/ k_{B} T\right)}+\text { offset }\right.}$ & $\sigma_{B C / T}^{+/ 0}=3(2) \times 10^{-8} \mathrm{~cm}^{2}$ \\
\hline & & $E_{B C / T}^{+/ 0}=0.38(1) \mathrm{eV}$ \\
\hline & & Offset $=3 \mathrm{MHz}$ \\
\hline \multicolumn{3}{|l|}{$\mathrm{Mu}_{T}^{0}$ relaxation } \\
\hline Spin exchange & $\Lambda_{T}^{0}=n v_{n} \sigma_{T}^{0}$ & $\sigma_{T}^{0}=2(2) \times 10^{-15} \mathrm{~cm}^{2}$ \\
\hline \multicolumn{3}{|l|}{ Site change } \\
\hline \multirow[t]{2}{*}{$\mathrm{Mu}_{T}^{0} \rightarrow \mathrm{Mu}_{B C}^{0}$} & $\Lambda_{T / B C}^{0 / 0}=\alpha_{T / B C}^{0 / 0} \exp ^{\left(-E_{T / B C}^{0 / 0} / k_{B} T\right)}$ & $\alpha_{T / B C}^{0 / 0}=2.5(5) \times 10^{7} \mathrm{MHz}$ \\
\hline & & $E_{T / B C}^{0 / 0}=0.38(1) \mathrm{eV}$ \\
\hline \multicolumn{3}{|l|}{ Charge exchange } \\
\hline \multirow[t]{2}{*}{$\mathrm{Mu}_{T}^{0}+h^{+} \rightarrow \mathrm{Mu}_{B C}^{+}$} & $\Lambda_{T / B C}^{0 /+}=p v_{p} \sigma_{T / B C}^{0 /+} \exp ^{\left(-E_{T / B C}^{0 /+} / k_{B} T\right)}$ & $\sigma_{T / B C}^{0 /+}=2.5(2) \times 10^{-11} \mathrm{~cm}^{2}$ \\
\hline & & $E_{T / B C}^{0 /+}=0.15(1) \mathrm{eV}$ \\
\hline
\end{tabular}

The prefactor and the activation energy are found to be 3.1 $\times 10^{7} \mathrm{MHz}$ and $0.21 \mathrm{eV}$, respectively; both are in agreement with values obtained previously from $\mathrm{rf}-\mu \mathrm{SR}$ experiments. ${ }^{8,28}$ Note that the direct site-change transition $\mathrm{Mu}_{B C}^{0} \rightarrow \mathrm{Mu}_{T}^{0}$ is deemed unlikely due to the large energy barrier involved. ${ }^{8}$ As a result, its contribution to the $\mathrm{Mu}_{B C}^{0}$ relaxation is omitted in the model (see Fig. 5). The optically induced relaxation rates of the $\mathrm{Mu}_{B C}^{0}$ signal at various temperatures are shown in Fig. 6(a). Clearly, the depolarization rate $\lambda_{B C}$ under illumination is increased significantly compared to the dark for most temperatures. Two additional processes involving $\mathrm{Mu}_{B C}^{0}$ become important: spin exchange processes ${ }^{11,26,29,30}$ with photogenerated electrons as well as capture processes with photogenerated holes $\left(\mathrm{Mu}_{B C}^{0}\right.$ $\left.+h^{+} \rightarrow \mathrm{Mu}_{B C}^{+}\right)$. These additional processes lead to depolarization of the $\mathrm{Mu}_{B C}^{0}$ signal even at low temperatures where thermal ionization of $\mathrm{Mu}_{B C}^{0}$ is not important. The spin exchange rate is set equal to $\Lambda_{B C}^{0}=n v_{n} \sigma_{B C}^{0}$, where $n$ is the net electron density (estimated to be $8 \times 10^{12} \mathrm{~cm}^{-3}$ under $100 \mathrm{~W}$ excitation $\left.^{38}\right), v_{n}$ is the electron thermal velocity, ${ }^{39}$ and $\sigma_{B C}^{0}$ is the scattering cross section. Similarly, the hole capture rate is set equal to $p v_{p} \sigma_{B C}^{0 /+}$, where $p$ is the hole carrier density (set equal to $n), v_{p}$ is the thermal velocity of holes, and $\sigma_{B C}^{0 /+}$ is the hole capture cross section. In the dark, below $\approx 125 \mathrm{~K}$, the relaxation associated with $\mathrm{Mu}_{B C}^{0}$ is small since the intrinsic concentrations of free carriers are small. The effects of spin exchange and hole capture processes are negligible here. However, under illumination, the free carrier concentrations increase dramatically and the relaxation rate is effectively increased by the amount $n v_{n} \sigma_{B C}^{0}+p v_{p} \sigma_{B C}^{0 /+}$, with cross sections found to be $\sigma_{B C}^{0}=2 \times 10^{-14} \mathrm{~cm}^{2}$ and $\sigma_{B C}^{0 /+}$

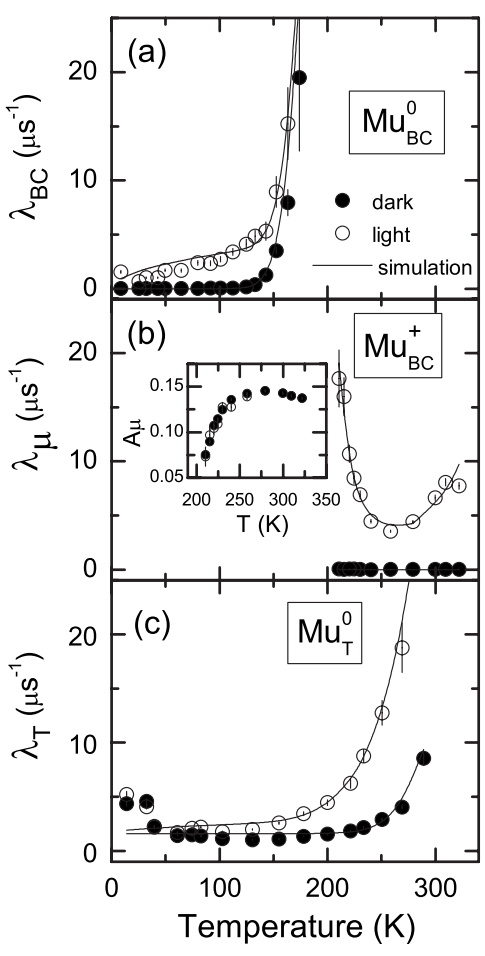

FIG. 6. Relaxation rates of the precession signals of (a) $\mathrm{Mu}_{B C}^{0}$, (b) $\mathrm{Mu}_{B C}^{+}$, and (c) $\mathrm{Mu}_{T}^{0}$. The open and solid circles are data in the dark and under illumination, respectively. The solid lines are the simulations using the three-state model and the parameters listed in Table. I. (The power of the lamp is 200,100 , and $80 \mathrm{~W}$ for $\mathrm{Mu}_{T}^{0}$, $\mathrm{Mu}_{B C}^{0}$, and $\mathrm{Mu}_{B C}^{+}$, respectively.) The inset in (b) shows the amplitude of the $\mathrm{Mu}_{B C}^{+}$signal as a function of temperature. 
$=4 \times 10^{-15} \mathrm{~cm}^{2}$. These cross sections are assumed to be independent of temperature. Our value of the hole capture cross section $\sigma_{B C}^{0 /+}$ is in reasonable agreement with the ones derived in Refs. 8 and 28. (It is notable that there is a slight temperature dependence of the $\mathrm{Mu}_{B C}^{0}$ relaxation rates below $\approx 125 \mathrm{~K}$, which may be dominated by the temperature dependence, i.e., $T^{1 / 2}$, of the carriers' velocities.)

Now, we turn to a discussion of the charged center $\mathrm{Mu}_{B C}^{+}$. Consistent with previous measurements, ${ }^{4}$ there is very little amplitude in the $\mathrm{Mu}_{B C}^{+}$precession signal below $200 \mathrm{~K}$; hence, they are only investigated at temperatures higher than $200 \mathrm{~K}$. (Recall that the majority of the implanted muons form either $\mathrm{Mu}_{B C}^{0}$ or $\mathrm{Mu}_{T}^{0}$ at low temperatures, ${ }^{4}$ and the transition of these states into $\mathrm{Mu}_{B C}^{+}$must be "rapid enough" at high temperatures for a coherent precession signal to be observed.) In the dark, there is very little relaxation of the $\mathrm{Mu}_{B C}^{+}$signal. By contrast, significant relaxation is observed upon illumination, as indicated in Figs. 2 and 6(b). We propose, as indicated in Fig. 5, that the generation of photoelectrons and the subsequent electron capture by $\mathrm{Mu}_{B C}^{+}$make the following transition channels relevant: (i) $\mathrm{Mu}_{B C}^{+}$ $+e^{-} \rightarrow \mathrm{Mu}_{B C}^{0}$, and (ii) $\mathrm{Mu}_{B C}^{+}+e^{-} \rightarrow \mathrm{Mu}_{T}^{0}$. The distinctive U-shaped temperature dependence for $\lambda_{\mu}$, shown in Fig. 6(b), can be qualitatively understood as follows: In the region from $\approx 200$ to $250 \mathrm{~K}$, there exists a considerable ionization of the $\mathrm{Mu}_{B C}^{0}$ to form $\mathrm{Mu}_{B C}^{+}$, as evidenced by the significant precession amplitude of $\mathrm{Mu}_{B C}^{+}$[see inset of Fig. 6(b)]. This $\Lambda_{B C}^{0 /+}$ conversion rate is significantly larger than the difference in precession frequencies associated with $\mathrm{Mu}_{B C}^{+}$and $\mathrm{Mu}_{B C}^{0}$. At the same time, the existence of the photoelectrons now produces a significant rate for the reverse conversion from $\mathrm{Mu}_{B C}^{+}$to $\mathrm{Mu}_{B C}^{0}$ due to electron capture. Hence, a cyclic charge exchange process develops $\left(\mathrm{Mu}_{B C}^{+} \leftrightarrow \mathrm{Mu}_{B C}^{0}\right)$. Since the transition rate $\Lambda_{B C}^{0 /+}$ is very fast, we are in the "dynamically narrowed" regime: increasing the temperature and, hence, $\Lambda_{B C}^{0 /+}$ leads to a decrease in the observed relaxation rate $\lambda_{\mu}$. Above $\approx 250 \mathrm{~K}$, another process becomes important in determining $\lambda_{\mu}$ : the conversion of $\mathrm{Mu}_{T}^{0}$ into $\mathrm{Mu}_{B C}^{+}$(see the discussion below for details on $\mathrm{Mu}_{T}^{0}$ ). This is evidenced by the increase in the dark values of $\lambda_{T}$ above $\approx 250 \mathrm{~K}$, as shown in Fig. 6(c). Thus, another charge exchange cycle $\left(\mathrm{Mu}_{B C}^{+} \leftrightarrow \mathrm{Mu}_{T}^{0}\right)$ develops. However, at these temperatures, the conversion rate $\Lambda_{T / B C}^{0 /+}$ from $\mathrm{Mu}_{T}^{0}$ to $\mathrm{Mu}_{B C}^{0}$ is still slower than the difference in precession frequencies between $\mathrm{Mu}_{T}^{0}$ and $\mathrm{Mu}_{B C}^{+}$. The system is thus in the "dynamically broadened" regime whereby increasing the temperature results in an increase in $\lambda_{\mu}$.

In order to obtain quantitative estimates of the transition rates, as listed in Table I, the electron capture process for $\mathrm{Mu}_{B C}^{+}+e^{-} \rightarrow \mathrm{Mu}_{B C}^{0}$ is modeled by the expression $\Lambda_{B C}^{+/ 0}$ $=n v_{n} \sigma_{B C}^{+/ 0}$, with the cross section $\sigma_{B C}^{+/ 0}$ fixed to the previously measured value of $3.3 \times 10^{-13} \mathrm{~cm}^{2} .28$ This value is roughly the correct order of magnitude expected for a Coulombic capture cross section between a positively charged particle $\mathrm{Mu}_{B C}^{+}$and a negatively charged electron. The process $\mathrm{Mu}_{B C}^{+}$ $+e^{-} \rightarrow \mathrm{Mu}_{T}^{0}$, which involves changes in both the charge state and site $(\mathrm{BC} \rightarrow T)$, is modeled using the approach adopted in Ref. 8. It is assumed this activated capture process takes the following form:

$$
n v_{n} \sigma_{B C / T}^{+/ 0} \exp ^{-\left(E_{B C / T}^{+/ 0} / k_{B} T\right)}
$$

The estimated values of the relevant parameters are $E_{B C / T}^{+/ 0}$ $=0.38 \mathrm{eV}$ and $\sigma_{B C / T}^{+/ 0}=3 \times 10^{-8} \mathrm{~cm}^{2}$. While our activation energy $E_{B C / T}^{+/ 0}$ agrees with the previously reported value, ${ }^{8,28}$ our activated cross section in this process is 2 orders of magnitude larger than the literature. The reason for this discrepancy is not well understood. It is worth noting that a small offset, assumed to be independent of temperature, is needed to fully model the $\lambda_{\mu}$ curve under illumination. This offset may suggest an additional capture process involving another diamagnetic state, e.g., $\mathrm{Mu}_{T}^{-}$, that is not explicitly taken into account in our model.

Finally, we discuss the fast diffusing neutral center $\mathrm{Mu}_{T}^{0}$. Figure 6(c) shows the temperature dependence of the $\mathrm{Mu}_{T}^{0}$ relaxation $\lambda_{T}$. In the dark at low temperatures, there is already a significant relaxation of the precession signal, which is contrary to the behavior of $\mathrm{Mu}_{B C}^{0}$ and $\mathrm{Mu}_{B C}^{+}$. The mechanism for such a "background" relaxation, which has been observed previously in unilluminated silicon, was suggested to be due to interactions of the mobile $\mathrm{Mu}_{T}^{0}$ with impurities in the material. ${ }^{4}$ As the temperature increases beyond $240 \mathrm{~K}$, the relaxation rates in the dark increase exponentially. This ionization process for $\mathrm{Mu}_{T}^{0}$ is now widely accepted to be a two step process: ${ }^{4,8}$ The $\mathrm{Mu}_{T}^{0}$ first makes a transition to $\mathrm{Mu}_{B C}^{0}$, where it is then converted into $\mathrm{Mu}_{B C}^{+}$. The $\mathrm{Mu}_{T}^{0} \rightarrow \mathrm{Mu}_{B C}^{0}$ transition can be described as an Arrhenius process

$$
\Lambda_{T / B C}^{0 / 0}=\alpha_{T / B C}^{0 / 0} \exp ^{\left(-E_{T / B C}^{0 / 0} k_{B} T\right)},
$$

and the subsequent $\mathrm{Mu}_{B C}^{0} \rightarrow \mathrm{Mu}_{B C}^{+}$transition has already been discussed above. The prefactor and the activation energy in the site-change process are found to be $2.5 \times 10^{7} \mathrm{MHz}$ and $0.38 \mathrm{eV}$, respectively; both are similar with values obtained previously from $\mathrm{rf}-\mu \mathrm{SR}$ experiments. ${ }^{8,28}$ Under illumination, we propose that two additional processes involving $\mathrm{Mu}_{T}^{0}$ become relevant: (1) the spin exchange and (2) the activated capture of holes to form $\mathrm{Mu}_{B C}^{+}$. The spin exchange transition, modeled by the rate $n v_{n} \sigma_{T}^{0}$, is the primary cause of the relaxation in the low temperature region up to $\approx 175 \mathrm{~K}$. In contrast to $\mathrm{Mu}_{B C}^{0}$, a simple Arrhenius relation is not sufficient to describe the high temperature relaxation when there is an abundance of photogenerated carriers available. Instead, there appears to be an activated capture process that starts becoming important at $\approx 175 \mathrm{~K}$. This is likely due to the hole capture of $\mathrm{Mu}_{T}^{0}$ into the $\mathrm{Mu}_{B C}^{+}$state. In analogy to the activated electron capture process discussed above for $\mathrm{Mu}_{B C}^{+}$, we model this rate as

$$
\Lambda_{T / B C}^{0 /+}=p v_{p} \sigma_{T / B C}^{0 /+} \exp ^{\left(-E_{T / B C}^{0 /+} / k_{B} T\right)}
$$

The values that best simulate the data are found to be $E_{T / B C}^{0 /+}=0.15 \mathrm{eV}$ and $\sigma_{T / B C}^{0 /+}=2.5 \times 10^{-11} \mathrm{~cm}^{2}$. The existence of such an activated hole capture process has also been 


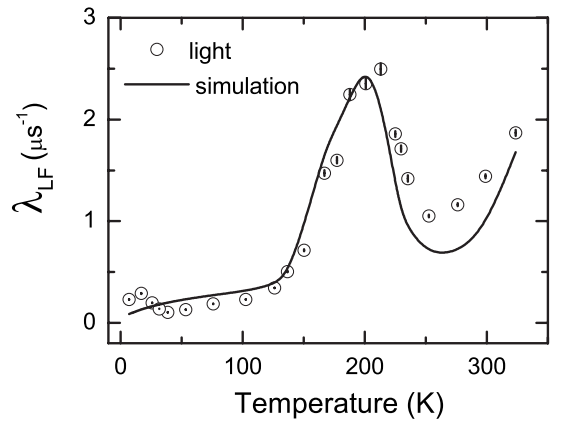

FIG. 7. Temperature dependence of the longitudinal field relaxation in an applied field of $0.1 \mathrm{~T}$ under illumination. The solid line represents the simulation using the model of Fig. 5 and the parameters in Table I.

suggested by a previous LF- $\mu$ SR photoexcitation experiment. ${ }^{29}$ However, no prefactor and activation energy parameters were deduced in this study.

The parameter values in Table I can also be used to simulate the temperature dependence of the longitudinal field relaxation $\lambda_{L F}$. The result is shown in Fig. 7 where the simulation is compared to the experimentally measured values of $\lambda_{L F}$ : The agreement is reasonable. Qualitatively, the depolarization at low temperatures (less than $125 \mathrm{~K}$ ) is dominated by the spin exchange of the neutral muonium states. The rapid rise at $140 \mathrm{~K}$ is predominantly due to the ionization (and recapture of photogenerated electrons) of the $\mathrm{Mu}_{B C}^{0}$. The peak temperature at $200 \mathrm{~K}$ is where the $\mathrm{Mu}_{B C}^{+}$enters the dynamically narrowed regime. Above $250 \mathrm{~K}$, the site change $T \rightarrow \mathrm{BC}$ followed by the fast ionization becomes large enough to be observed.

Hence, our current results qualitatively support the general conclusions of previous photoexcitation experiments in silicon; i.e., muonium undergoes significant interactions with photogenerated electrons and holes. In addition, by making use of our ability to study the unique precession signatures associated with the three muonium states, the present work also provides more quantitative information on the parameters characterizing these photoinduced dynamics.

Finally, we note that previous LF- $\mu$ SR photoexcitation studies on intermediate doped $p$-type and $n$-type Si (Ref. 29) as well as doped Ge (Ref. 37) have found evidence for a delayed formation of electrically inactive muonium. No clear evidence of such behavior is found in our current study of high resistivity $\mathrm{Si}$. It may be interesting to apply our technique to investigate more heavily doped $\mathrm{Si}$ and $\mathrm{Ge}$ as well.

\section{CONCLUSION}

In conclusion, we have demonstrated that optical excitation can dramatically enhance the relaxation of the precession signatures associated with $\mathrm{Mu}_{B C}^{+}, \mathrm{Mu}_{B C}^{0}$, and $\mathrm{Mu}_{T}^{0}$ in high resistivity silicon, hence providing detailed information on the dynamics, such as activation energies and capture cross sections, of the three major muonium states. A threestate model previously suggested to explain the radiofrequency data in unilluminated $\mathrm{Si}$ is a good description of the temperature dependence of the relaxation rates both in
TF and LF modes, provided that the effects of photogenerated electrons and holes are taken into account in the spin exchange and carrier capture processes.

\section{ACKNOWLEDGMENTS}

This work is supported by the Natural Sciences and Engineering Research Council (NSERC), R.A. Welch Foundation, and the U.S. National Science Foundation. We would like to thank R. F. Kiefl, R. Kadono, and K. Shimomura for helpful discussions in the initial stages of this research.

\section{APPENDIX: MODELING THE NET POLARIZATION}

The calculation of the time-dependent muon polarization can be accomplished by using the formalism first described by Odermatt. ${ }^{36}$ In short, the density matrix of a single muonium state, consisting of a muon and an electron, leads to a set of 15 coupled differential equations, which may be written compactly as

$$
\frac{d \mathbf{P}}{d t}=\hat{M} \mathbf{P} .
$$

The polarization $\mathbf{P}$ is represented by a $15 \times 1$ vector that describes the state of the muon, its bound electron (in the case of neutral muonium), and the muon-electron interactions. In particular, the first three elements of $\mathbf{P}$ give the $x, y$, and $z$ components of the muon polarization. The matrix $\hat{M}$ has a $15 \times 15$ dimension and is derived from the coupled differential equations; its matrix elements can be found in Ref. 36. The solution to Eq. (A1) is often expressed as

$$
\mathbf{P}=\sum_{i=1}^{15} c_{i} \mathbf{a}_{i} e^{\left(-\alpha_{i} t\right)}
$$

where the parameters $\mathbf{a}_{i}$ and $\alpha_{i}$ are the eigenvectors and (complex) eigenvalues of the matrix $\hat{M}$, respectively. The coefficient $c_{i}$ is determined from the initial polarization at $t$ $=0$. The effect of the spin exchange of neutral muonium is taken into account by subtracting $2 \Lambda^{0}$ (the spin exchange rate) from the terms involving the bound electrons in the coefficient matrix $\hat{M}$.

In our model, there can be up to three muonium centers coexisting in the sample. Each of these centers can (in principle) make a transition to any of the other two centers. If the three centers are labeled as $\mathrm{BC}\left(\mathrm{Mu}_{B C}^{0}\right), \mu\left(\mathrm{Mu}^{+}\right)$, and $T$ $\left(\mathrm{Mu}_{T}^{0}\right)$, then Eq. (A1) is still formally correct, but with terms that should be interpreted as follows: ${ }^{36}$

$$
\mathbf{P}=\left(\begin{array}{c}
\mathbf{P}_{B C} \\
\mathbf{P}_{\mu} \\
\mathbf{P}_{T}
\end{array}\right)
$$




$$
\hat{M}=\left(\begin{array}{ccc}
\hat{M}_{B C}-\left(\Lambda_{B C}^{0 /+}+\Lambda_{B C / T}^{0 / 0}\right) I & \Lambda_{B C}^{+/ 0} A & \Lambda_{T / B C}^{0 / 0} A \\
\Lambda_{B C}^{0 /+} A & \hat{M}_{\mu}-\left(\Lambda_{B C}^{+/ 0}+\Lambda_{B C / T}^{+/ 0}\right) I & \Lambda_{T / B C}^{0++} A \\
\Lambda_{B C / T}^{0 / 0} A & \Lambda_{B C / T}^{+/ 0} A & \hat{M}_{T}-\left(\Lambda_{T / B C}^{0 / 0}+\Lambda_{T / B C}^{0 /+}\right) I
\end{array}\right)
$$

The term $\mathbf{P}$ is now a $45 \times 1$ vector that describes the polarizations of the three muon states simultaneously while the coefficient matrix $\hat{M}$ is a $45 \times 45$ matrix that contains the $15 \times 15$ matrices describing each isolated center (i.e., $\hat{M}_{B C}$, $\hat{M}_{\mu}, \hat{M}_{T}$ ). The notation for the transition rates in Eq. (A4) is the same as in Fig. 5. Rates located in the diagonal positions of Eq. (A4) are multiplied with a $15 \times 15$ identity matrix $I$.
The off-diagonal elements are multiplied by a $15 \times 15$ matrix $A$. Matrix $A$ is the same as the identity matrix if the electron polarizations are conserved during the transitions. However, if the electron polarizations are assumed to be lost during transitions, as in the case of thermal ionization of neutral centers into charged states, the diagonal elements of $A$ involving electrons have to be zeroed $\left(A_{i i}=0\right.$ for $\left.i=4-15\right)$. *ifan@phys.ualberta.ca

†kimchow@phys.ualberta.ca

${ }^{1}$ S. M. Myers, M. I. Baskes, H. K. Birnbaum, J. W. Corbett, G. G. DeLeo, S. K. Estreicher, E. E. Haller, P. Jena, N. M. Johnson, R. Kirchheim, S. J. Pearton, and M. J. Stavola, Rev. Mod. Phys. 64, 559 (1992).

${ }^{2}$ J. I. Pankove and N. M. Johnson, Semicond. Semimetals 34, 1 (1991).

${ }^{3}$ C. G. Van de Walle and J. Neugebauer, Nature (London) 423, 626 (2003).

${ }^{4}$ B. D. Patterson, Rev. Mod. Phys. 60, 69 (1988).

${ }^{5}$ K. H. Chow, B. Hitti, and R. F. Kiefl, Semicond. Semimetals 51A, 137 (1998).

${ }^{6}$ H. Simmler, P. Eschle, H. Keller, W. Kundig, W. Odermatt, B. D. Patterson, I. M. Savic, J. W. Schneider, B. Staublepumpin, U. Straumann, and P. Truol, Nucl. Instrum. Methods Phys. Res. B 63, 125 (1992).

${ }^{7}$ R. Kadono, A. Matsushita, K. Nagamine, K. Nishiyama, K. H. Chow, R. F. Kiefl, A. MacFarlane, D. Schumann, S. Fujii, and S. Tanigawa, Phys. Rev. B 50, 1999 (1994).

${ }^{8}$ S. R. Kreitzman, B. Hitti, R. L. Lichti, T. L. Estle, and K. H. Chow, Phys. Rev. B 51, 13117 (1995).

${ }^{9}$ K. H. Chow, R. F. Kiefl, W. A. MacFarlane, J. W. Schneider, D. W. Cooke, M. Leon, M. Paciotti, T. L. Estle, B. Hitti, R. L. Lichti, S. F. J. Cox, C. Schwab, E. A. Davis, A. Morrobel-Sosa, and L. Zavieh, Phys. Rev. B 51, 14762 (1995).

${ }^{10}$ K. H. Chow, B. Hitti, R. F. Kiefl, S. R. Dunsiger, R. L. Lichti, and T. L. Estle, Phys. Rev. Lett. 76, 3790 (1996).

${ }^{11}$ K. H. Chow, R. F. Kiefl, B. Hitti, T. L. Estle, and R. L. Lichti, Phys. Rev. Lett. 84, 2251 (2000).

${ }^{12}$ K. H. Chow, Physica B 326, 145 (2003).

${ }^{13}$ B. E. Schultz, K. H. Chow, B. Hitti, Z. Salman, S. R. Kreitzman, R. F. Kiefl, and R. L. Lichti, Phys. Rev. B 72, 033201 (2005).

${ }^{14}$ B. E. Schultz, K. H. Chow, B. Hitti, R. F. Kiefl, R. L. Lichti, and S. F. J. Cox, Phys. Rev. Lett. 95, 086404 (2005).

${ }^{15}$ K. H. Chow, B. Hitti, and J. S. Lord, Phys. Rev. B 73, 113202 (2006).

${ }^{16}$ Z. Salman, T. J. Parolin, K. H. Chow, T. A. Keeler, R. I. Miller,
D. Wang, and W. A. MacFarlane, Phys. Rev. B 73, 174427 (2006).

${ }^{17}$ R. L. Lichti, S. F. J. Cox, K. H. Chow, E. A. Davis, T. L. Estle, B. Hitti, E. Mytilineou, and C. Schwab, Phys. Rev. B 60, 1734 (1999).

${ }^{18}$ K. H. Chow, B. Hitti, R. F. Kiefl, R. L. Lichti, and T. L. Estle, Phys. Rev. Lett. 87, 216403 (2001).

${ }^{19}$ S. F. J. Cox, J. Phys.: Condens. Matter 15, R1727 (2003).

${ }^{20}$ K. Shimomura, R. Kadono, K. Ohishi, M. Mizuta, M. Saito, K. H. Chow, B. Hitti, and R. L. Lichti, Phys. Rev. Lett. 92, 135505 (2004).

${ }^{21}$ R. L. Lichti, W. A. Nussbaum, and K. H. Chow, Phys. Rev. B 70, 165204 (2004).

${ }^{22}$ R. L. Lichti, H. N. Bani-Salameh, B. R. Carroll, K. H. Chow, B. Hitti, and S. R. Kreitzman, Phys. Rev. B 76, 045221 (2007).

${ }^{23}$ R. C. Vilao, H. V. Alberto, J. P. Duarte, J. M. Gil, A. Weidinger, N. Ayres de Campos, R. L. Lichti, K. H. Chow, and S. F. J. Cox, Phys. Rev. B 72, 235203 (2005).

${ }^{24}$ R. F. Kiefl, M. Celio, T. L. Estle, S. R. Kreitzman, G. M. Luke, T. M. Riseman, and E. J. Ansaldo, Phys. Rev. Lett. 60, 224 (1988).

${ }^{25}$ J. W. Schneider, K. Chow, R. F. Kiefl, S. R. Kreitzman, A. MacFarlane, R. C. DuVarney, T. L. Estle, R. L. Lichti, and C. Schwab, Phys. Rev. B 47, 10193 (1993).

${ }^{26}$ K. H. Chow, R. L. Lichti, R. F. Kiefl, S. Dunsiger, T. L. Estle, B. Hitti, R. Kadono, W. A. MacFarlane, J. W. Schneider, D. Schumann, and M. Shelley, Phys. Rev. B 50, 8918 (1994).

${ }^{27}$ K. H. Chow, R. F. Kiefl, J. W. Schneider, B. Hitti, T. L. Estle, R. L. Lichti, C. Schwab, R. C. DuVarney, S. R. Kreitzman, W. A. MacFarlane, and M. Senba, Phys. Rev. B 47, 16004 (1993).

${ }^{28}$ B. Hitti, S. R. Kreitzman, T. L. Estle, E. S. Bates, M. R. Dawdy, T. L. Head, and R. L. Lichti, Phys. Rev. B 59, 4918 (1999).

${ }^{29}$ R. Kadono, R. M. Macrae, and K. Nagamine, Phys. Rev. B 68, 245204 (2003).

${ }^{30}$ R. Kadono, A. Matsushita, R. M. Macrae, K. Nishiyama, and K. Nagamine, Phys. Rev. Lett. 73, 2724 (1994).

${ }^{31}$ M. Iwanowski, K. Maier, J. Major, Th. Pfiz, R. Scheuermann, L. Schimmele, A. Seeger, and M. Hampele, Hyperfine Interact. 86, 681 (1994). 
${ }^{32}$ R. Scheuermann, J. Major, A. Seeger, L. Schimmele, J. Schmidl, and D. Herlach, Physica B 289-290, 534 (2000).

${ }^{33}$ G. D. Morris, W. A. MacFarlane, K. H. Chow, Z. Salman, D. J. Arseneau, S. Daviel, A. Hatakeyama, S. R. Kreitzman, C. D. P. Levy, R. Poutissou, R. H. Heffner, J. E. Elenewski, L. H. Greene, and R. H. Kiefl, Phys. Rev. Lett. 93, 157601 (2004); Z. Salman, A. I. Mansour, K. H. Chow, M. Beaudoin, I. Fan, J. Jung, T. A. Keeler, R. F. Kiefl, C. D. P. Levy, R. C. Ma, G. D. Morris, T. J. Parolin, D. Wang, and W. A. MacFarlane, Phys. Rev. B 75, 073405 (2007); T. J. Parolin, Z. Salman, J. Chakhalian, Q. Song, K. H. Chow, M. D. Hossain, T. A. Keeler, R. F. Kiefl, S. R. Kreitzman, C. D. P. Levy, R. I. Miller, G. D. Morris, M. R. Pearson, H. Saadaoui, D. Wang, and W. A. MacFarlane, Phys. Rev. Lett. 98, 047601 (2007); Z. Salman, D. Wang, K. H. Chow, M. D. Hossain, S. Kreitzman, T. A. Keeler, C. D. P. Levy, W. A. MacFarlane, R. I. Miller, G. D. Morris, T. J. Parolin, H. Saadaoui, M. Smadella, and R. F. Kiefl, ibid. 98, 167001 (2007); Z. Salman, K. H. Chow, R. I. Miller, A. Morello, T. J. Parolin, M. D. Hossain, T. A. Keeler, C. D. P. Levy, W. A. MacFarlane, G. D. Morris, H. Saadaoui, D. Wang, R. Sessoli, G. G. Condorelli, and R. F. Kiefl, Nano Lett. 7, 1551 (2007); M. Xu, M. D. Hossain, H. Saadaoui, T. J. Parolin, K. H. Chow, T. A. Keeler, R. F. Kiefl, G. D. Moris, Z. Salman, Q. Song, D. Wang, and W. A. MacFarlane, J. Mater. Res. (to be published).
${ }^{34}$ Z. Salman, E. P. Reynard, W. A. MacFarlane, K. H. Chow, J. Chakhalian, S. R. Kreitzman, S. Daviel, C. D. P. Levy, R. Poutissou, and R. F. Kiefl, Phys. Rev. B 70, 104404 (2004); Z. Salman, R. F. Kiefl, K. H. Chow, M. D. Hossain, T. A. Keeler, S. R. Kreitzman, C. D. P. Levy, R. I. Miller, T. J. Parolin, M. R. Pearson, H. Saadaoui, J. D. Schultz, M. Smadella, D. Wang, and W. A. MacFarlane, Phys. Rev. Lett. 96, 147601 (2006).

${ }^{35}$ A. Hintermann, P. F. Meier, and B. D. Patterson, Am. J. Phys. 48, 956 (1980).

${ }^{36}$ W. Odermatt, Helv. Phys. Acta 61, 1087 (1988).

${ }^{37}$ R. Kadono, R. M. Macrae, K. Nishiyama, and K. Nagamine, Phys. Rev. B 55, 4035 (1997).

${ }^{38}$ The carrier density $n$ is a sum of the intrinsic carriers and extrinsic carriers. The extrinsic carriers are generated by the optical excitation and is significantly larger than the intrinsic carrier density. The extrinsic carrier density is estimated by fitting the product $n v_{n} \sigma_{B C}^{+/ 0}$ to the $\mathrm{Mu}_{B C}^{+}$relaxation while fixing the cross section to $3.3 \times 10^{-13} \mathrm{~cm}^{-2}$. In addition, because the intensity of the optical excitation used in the experiments is different for each of the three muonium centers, a different scaling multiplier is applied to the carrier density during the simulations of different muonium centers.

${ }^{39}$ The thermal velocity of electrons (or holes) can be calculated using the equipartition theorem, which yields $v_{e}=\sqrt{3 k_{B} T / m^{*}}$, where $m^{*}$ is the effective mass of carriers in silicon. 